DPNU-03-26

November 3, 2018

\title{
Chiral Doubling of Heavy-Light Hadrons and the Vector Manifestation of Hidden Local Symmetry
}

\author{
Masayasu Harada, ${ }^{1}$ Mannque Rho, ${ }^{2,3}$ and Chihiro Sasaki ${ }^{1}$ \\ ${ }^{1}$ Department of Physics, Nagoya University, Nagoya, 464-8602, Japan \\ ${ }^{2}$ Service de Physique Théorique, CEA Saclay, 91191 Gif-sur-Yvette, France \\ ${ }^{3}$ Department of Physics, Hanyang University, Seoul 133-791, Korea
}

\begin{abstract}
Starting with a hidden local symmetry Lagrangian at the vector manifestation (VM) fixed point that incorporates heavy-quark symmetry and matching the bare theory to QCD, we calculate the splitting of chiral doublers of heavy-light mesons proposed by Nowak, Rho and Zahed [1] and Bardeen and Hill 2]. We show, in the three-flavor chiral limit, that the splitting is directly proportional to the light-quark condensate $\langle\bar{q} q\rangle$ and comes out to be $\sim \frac{1}{3} m_{N}$ where $m_{N}$ is the nucleon mass, implying that the splitting vanishes in the chiral limit at the chiral restoration point - temperature $T_{c}$, density $n_{c}$ or number of flavors $N_{F}^{c}$. The result turns out to be surprisingly simple with the vector $(\rho)$ meson playing the crucial role in quantum corrections, pointing to the relevance of the VM to QCD in the way chiral symmetry is manifested in hadronic matter. We also make predictions on the hadronic decay processes of the excited heavy (charm) -light mesons $\tilde{D}$.
\end{abstract}




\section{INTRODUCTION}

Based on the manifestation of chiral symmetry à la linear sigma model, it was predicted a decade ago [1, 2] that the mass splitting $\Delta M$ between the $\mathcal{M}\left(0^{-}, 1^{-}\right)$and $\tilde{\mathcal{M}}\left(0^{+}, 1^{+}\right)$mesons where $\mathcal{M}$ denotes a heavy-light meson consisting of heavy qaurk $Q$ and light antiquark $\bar{q}$ should be of the size of the constituent quark mass. Recently, BaBar [3], CLEO [4] and subsequently the Belle collaboration [5], discovered new $D$ mesons with $Q=c, c$ being charm quark, which most likely have spin-party $0^{+}$and $1^{+}$and the mass difference to the $D\left(0^{-}, 1^{-}\right)$is in fair agreement with the prediction of [1, 2]. In a recent article, Nowak, Rho and Zahed [6] proposed that the splitting of $\mathcal{M}$ and $\tilde{\mathcal{M}}$ mesons could carry direct information on the property of chiral symmetry at some critical density or temperature at which the symmetry is restored \#1. In this paper we pick up this idea and make a first step in consolidating the proposal of Ref. [6]. In doing this, we shall take the reverse direction: Instead of starting with a Lagrangian defined in the chiral-symmetry broken phase and then driving the system to the chiral symmetry restoration point by an external disturbance, we will start from an assumed structure of chiral symmetry at its restoration point and then make a prediction as to what happens to the splitting in the broken phase. We find that the splitting is directly proportional to the lightquark condensate and comes out to be of the size of the constituent quark mass consistent with the prediction of Refs. 1, 2]. We shall associate this result as giving a link between the assumed structure of the chiral restoration point and the broken phase.

Our procedure is based on the result that the effective field theory (EFT) implementing hidden local symmetry (HLS) 7, 8], when matched to QCD at a suitable matching scale $\Lambda_{M}$, represents QCD up to the matching scale [9, 10] and the "vector manifestation" (VM) [9, 11] is realized in the chiral limit at the chiral restoration point generically denoted $C_{\chi}$ (critical temperature $T_{c}$ [12] or density $n_{c}$ [13] or number of flavors $N_{F}^{c}$ [11]). In the HLS theory consisting of pions and vector mesons, the VM is characterized by the existence of a fixed point called VM fixed point at which the HLS gauge coupling constant $g$ and the vector meson mass $m_{V}$ vanish with the longitudinal components of the vector mesons joining in the multiplet with the pions and the pion decay constant $f_{\pi} \rightarrow 0$. In this theory - referred to in short as HLS/VM, the system flows uniquely to the fixed point as one approaches $C_{\chi}$ from below. The VM fixed point implies that light vector meson masses vanish proportionally to the quark condensate $\langle\bar{q} q\rangle$ - the order parameter of chiral symmetry - as one approaches $C_{\chi}$, supporting the scenario suggested in BR scaling [14]. We assume that the heavy-light hadrons

\footnotetext{
${ }^{\# 1}$ In what follows, unless otherwise specified, we will refer to heavy-light mesons generically as $D$ but the arguments should apply better to heavier-quark mesons. Numerical estimates will however be made solely for the (open charm) $D$ mesons.
} 
are described by a VM-fixed point theory at $C_{\chi}$ and by introducing the simplest form of the VM breaking terms, we compute the mass splitting of the chiral doublers in matter-free space in terms of the quantities that figure in the QCD correlators $\# 2$.

Before going into our main theme, we should note that the presence of light vector mesons near the VM fixed point makes certain predictions that are basically different from the standard scenario in which the only relevant (hadronic) degrees of freedom near the critical point are the pions (and a light scalar). For instance, the HLS/VM [9] predicts that the pion velocity approaches the speed of light as $T \rightarrow T_{c}$ [15] in a stark contrast to the standard picture where the pion velocity goes to zero [16]. Whether or not the light vectors do actually figure importantly in the vicinity of the chiral phase transition should ultimately be checked by lattice calculations. At the moment, there is no clear evidence either for or against the VM scenario: What is needed but not yet available is measurement of dynamical correlation functions. The forthcoming "maximum entropy method (MEM)" analysis [17] for excitations just below $T_{c}$ might shed light on this important issue. In this paper, we shall simply assume that the chiral restoration is described by HLS/VM and ask whether this assumption is consistent with the splitting observed by BaBar, CLEO and Belle.

This paper is organized as follows: In section 2, we write down the EFT Lagrangian that defines our approach. In section 3 we perform the matching to determine the bare parameters of the EFT Lagrangian. Section 4 is devoted to computing the quantum correction to the mass splitting and obtaining the renormalization group equation for the parameter expressing the splitting. In section 5, we give a semi-quantitative estimate of the value of the mass splitting. To see whether or not our scenario based on the VM differs from that à la linear sigma model, we study the consequences of our scenario on the hadronic decay processes of the open charm $\tilde{D}$ meson in section 6. We give a brief summary and discussions in section 7

\section{LAGRANGIAN}

In this section we give our reasoning that leads to the Lagrangian that defines our approach. Here we construct the Lagrangian using the approximate chiral $S U(3)_{\mathrm{L}} \times S U(3)_{\mathrm{R}}$ symmetry in the light-quark sector and the heavy quark symmetry in the heavy-quark sector. We will start from the Lagrangian given at the vector manifestation (VM) fixed point. We first describe how to construct the fixed point Lagrangian based on the VM. Then, we account for the effect of spontaneous chiral symmetry breaking by adding a bare parameter for the mass splitting

\#2 Introducing vector mesons in the light-quark sector of heavy-light mesons was considered in Ref. [1] but without the matching to QCD and hence without the VM fixed point. 
in the heavy sector and including the deviation of the HLS parameters from the values at the VM fixed point. The explicit form of the Lagrangian so constructed is shown in subsection 2.3.

\subsection{The fixed point Lagrangian}

To define the notations, we briefly review the model based on the hidden local symmetry (HLS) 7, 8]. The HLS model $\# 3$ is based on the $G_{\text {global }} \times H_{\text {local }}$ symmetry, where $G=$ $S U(3)_{\mathrm{L}} \times S U(3)_{\mathrm{R}}$ is the chiral symmetry and $H=S U(3)_{\mathrm{V}}$ is the HLS. The basic quantities are the HLS gauge boson and two matrix valued variables $\xi_{\mathrm{L}}(x)$ and $\xi_{\mathrm{R}}(x)$ which transform as

$$
\xi_{\mathrm{L}, \mathrm{R}}(x) \rightarrow \xi_{\mathrm{L}, \mathrm{R}}^{\prime}(x)=h(x) \cdot \xi_{\mathrm{L}, \mathrm{R}}(x) \cdot g_{\mathrm{L}, \mathrm{R}}^{\dagger}
$$

where $h(x) \in H_{\text {local }}$ and $g_{L, R} \in\left[S U(3)_{\mathrm{L}, \mathrm{R}}\right]_{\text {global }}$. These variables are parameterized as

$$
\xi_{\mathrm{L}, \mathrm{R}}(x)=e^{i \sigma(x) / F_{\sigma}} e^{\mp i \pi(x) / F_{\pi}}
$$

where $\pi=\pi^{a} T_{a}$ denotes the pseudoscalar Nambu-Goldstone bosons associated with the spontaneous symmetry breaking of $G_{\text {global }}$ chiral symmetry, and $\sigma=\sigma^{a} T_{a}$ denotes the NambuGoldstone bosons associated with the spontaneous breaking of $H_{\text {local }}$. This $\sigma$ is eaten up by the HLS gauge boson becoming massive through the Higgs mechanism. $F_{\pi}$ and $F_{\sigma}$ are the decay constants of the associated particles. The phenomenologically important parameter $a$ is defined by the ratio

$$
a=\frac{F_{\sigma}^{2}}{F_{\pi}^{2}} .
$$

The covariant derivatives of $\xi_{\mathrm{L}, \mathrm{R}}$ are given by

$$
\begin{aligned}
& D_{\mu} \xi_{\mathrm{L}}=\partial_{\mu} \xi_{\mathrm{L}}-i V_{\mu} \xi_{\mathrm{L}} \\
& D_{\mu} \xi_{\mathrm{R}}=\partial_{\mu} \xi_{\mathrm{R}}-i V_{\mu} \xi_{\mathrm{R}}
\end{aligned}
$$

where $V_{\mu}$ is the gauge field of $H_{\text {local }}$.

The basic quantities in constructing the Lagrangian are the following two 1-forms:

$$
\begin{aligned}
& \hat{\alpha}_{\| \mu}=\frac{1}{2 i}\left(D_{\mu} \xi_{\mathrm{R}} \cdot \xi_{\mathrm{R}}^{\dagger}+D_{\mu} \xi_{\mathrm{L}} \cdot \xi_{\mathrm{L}}^{\dagger}\right) \\
& \hat{\alpha}_{\perp \mu}=\frac{1}{2 i}\left(D_{\mu} \xi_{\mathrm{R}} \cdot \xi_{\mathrm{R}}^{\dagger}-D_{\mu} \xi_{\mathrm{L}} \cdot \xi_{\mathrm{L}}^{\dagger}\right) .
\end{aligned}
$$

\footnotetext{
\#3 In the modern interpretation [18], implementing HLS in the chiral Lagrangian can be associated with the "ultraviolet completion" to the fundamental theory of strong interactions, i.e., QCD. The matching to QCD at a matching scale is therefore a crucial ingredient of the approach.
} 
They transform as

$$
\hat{\alpha}_{\perp, \|}^{\mu} \rightarrow h(x) \cdot \hat{\alpha}_{\perp, \|}^{\mu} \cdot h^{\dagger}(x)
$$

When HLS is gauge-fixed to the unitary gauge, $\sigma=0$, $\xi_{\mathrm{L}}$ and $\xi_{\mathrm{R}}$ are related with each other by

$$
\xi_{\mathrm{L}}^{\dagger}=\xi_{\mathrm{R}} \equiv \xi=e^{i \pi / F_{\pi}}
$$

This unitary gauge is not preserved under the $G_{\text {global }}$ transformation, which in general has the following form

$$
\begin{aligned}
G_{\text {global }}: \xi \rightarrow \xi^{\prime} & =\xi \cdot g_{\mathrm{R}}^{\dagger}=g_{\mathrm{L}} \cdot \xi \\
& =\exp \left[i \sigma^{\prime}\left(\pi, g_{\mathrm{R}}, g_{\mathrm{L}}\right) / F_{\sigma}\right] \exp \left[i \pi^{\prime} / F_{\pi}\right] \\
& =\exp \left[i \pi^{\prime} / F_{\pi}\right] \exp \left[-i \sigma^{\prime}\left(\pi, g_{\mathrm{R}}, g_{\mathrm{L}}\right) / F_{\sigma}\right]
\end{aligned}
$$

The unwanted factor $\exp \left[i \sigma^{\prime}\left(\pi, g_{\mathrm{R}}, g_{\mathrm{L}}\right) / F_{\sigma}\right]$ can be eliminated if we simultaneously perform the $H_{\text {local }}$ gauge transformation with

$$
H_{\text {local }}: h=\exp \left[i \sigma^{\prime}\left(\pi, g_{\mathrm{R}}, g_{\mathrm{L}}\right) / F_{\sigma}\right] \equiv h\left(\pi, g_{\mathrm{R}}, g_{\mathrm{L}}\right)
$$

Then the system has a global symmetry $G=S U(3)_{\mathrm{L}} \times S U(3)_{\mathrm{R}}$ under the following combined transformation:

$$
G: \xi \rightarrow h\left(\pi, g_{\mathrm{R}}, g_{\mathrm{L}}\right) \cdot \xi \cdot g_{\mathrm{R}}^{\dagger}=g_{\mathrm{L}} \cdot \xi \cdot h\left(\pi, g_{\mathrm{R}}, g_{\mathrm{L}}\right)
$$

Under this transformation the HLS gauge boson field $V_{\mu}$ in the unitary gauge transforms as

$$
G: V_{\mu} \rightarrow h\left(\pi, g_{\mathrm{R}}, g_{\mathrm{L}}\right) \cdot V_{\mu} \cdot h^{\dagger}\left(\pi, g_{\mathrm{R}}, g_{\mathrm{L}}\right)-i \partial h\left(\pi, g_{\mathrm{R}}, g_{\mathrm{L}}\right) \cdot h^{\dagger}\left(\pi, g_{\mathrm{R}}, g_{\mathrm{L}}\right)
$$

which is nothing but the transformation property of Weinberg's " $\rho$ meson" [19]. The two 1-forms $\hat{\alpha}_{\|}^{\mu}$ and $\hat{\alpha}_{\perp}^{\mu}$ transform as

$$
\hat{\alpha}_{\perp, \|}^{\mu} \rightarrow h\left(\pi, g_{\mathrm{R}}, g_{\mathrm{L}}\right) \cdot \hat{\alpha}_{\perp, \|}^{\mu} \cdot h^{\dagger}\left(\pi, g_{\mathrm{R}}, g_{\mathrm{L}}\right)
$$

Then, we can regard these 1 -forms as the fields belonging to the chiral representations $(1,8)+$ $(8,1)$ and $(1,8)-(8,1)$ under $S U(3)_{\mathrm{L}} \times S U(3)_{\mathrm{R}}$.

Let us next consider the VM at the point at which chiral symmetry is restored (in the chiral limit). At the VM at its fixed point characterized by $(g, a)=(0,1)$, the two 1 -forms become

$$
\begin{aligned}
& \alpha_{\| \mu}=\frac{1}{2 i}\left(\partial_{\mu} \xi_{\mathrm{R}} \cdot \xi_{\mathrm{R}}^{\dagger}+\partial_{\mu} \xi_{\mathrm{L}} \cdot \xi_{\mathrm{L}}^{\dagger}\right) \\
& \alpha_{\perp \mu}=\frac{1}{2 i}\left(\partial_{\mu} \xi_{\mathrm{R}} \cdot \xi_{\mathrm{R}}^{\dagger}-\partial_{\mu} \xi_{\mathrm{L}} \cdot \xi_{\mathrm{L}}^{\dagger}\right) .
\end{aligned}
$$


Note that the above $\alpha_{\| \mu}$ and $\alpha_{\perp \mu}$ do not contain the HLS gauge field since the gauge coupling $g$ vanishes at the VM fixed point. It is convenient to define the (L,R) 1-forms:

$$
\begin{gathered}
\alpha_{R \mu}=\alpha_{\| \mu}+\alpha_{\perp \mu}=\frac{1}{i} \partial_{\mu} \xi_{\mathrm{R}} \cdot \xi_{\mathrm{R}}^{\dagger}, \\
\alpha_{L \mu}=\alpha_{\| \mu}-\alpha_{\perp \mu}=\frac{1}{i} \partial_{\mu} \xi_{\mathrm{L}} \cdot \xi_{\mathrm{L}}^{\dagger},
\end{gathered}
$$

which can be regarded as belonging to the chiral representation $(1,8)$ and $(8,1)$, respectively, transforming under chiral $S U(3)_{\mathrm{L}} \times S U(3)_{\mathrm{R}}$ as

$$
\begin{aligned}
\alpha_{R \mu} & \rightarrow g_{\mathrm{R}} \alpha_{R \mu} g_{\mathrm{R}}^{\dagger}, \\
\alpha_{L \mu} & \rightarrow g_{\mathrm{L}} \alpha_{L \mu} g_{\mathrm{L}}^{\dagger} .
\end{aligned}
$$

By using these 1-forms, the HLS Lagrangian at the VM fixed point can be written as $[9]$

$$
\mathcal{L}_{\text {light }}^{*}=\frac{1}{2} F_{\pi}^{2} \operatorname{tr}\left[\alpha_{R \mu} \alpha_{R}^{\mu}\right]+\frac{1}{2} F_{\pi}^{2} \operatorname{tr}\left[\alpha_{L \mu} \alpha_{L}^{\mu}\right],
$$

where the $*$ affixed to the Lagrangian denotes that it is a fixed-point Lagrangian, and $F_{\pi}$ denotes the bare pion decay constant. Note that the physical pion decay constant $f_{\pi}$ vanishes at the VM fixed point by the quadratic divergence although the bare one is non-zero [9]. It should be stressed that the above fixed point Lagrangian is approached only as a limit of chiral symmetry restoration $[9]$.

Next we consider the fixed-point Lagrangian of the heavy meson sector at the chiral restoration point identified with the VM fixed point. Let us introduce two heavy-meson fields $\mathcal{H}_{R}$ and $\mathcal{H}_{L}$ transforming under chiral $\mathrm{SU}(3)_{\mathrm{R}} \times \mathrm{SU}(3)_{\mathrm{L}}$ as

$$
\mathcal{H}_{R} \rightarrow \mathcal{H}_{R} g_{\mathrm{R}}^{\dagger}, \quad \mathcal{H}_{L} \rightarrow \mathcal{H}_{L} g_{\mathrm{L}}^{\dagger} .
$$

By using these fields together with the light-meson 1-forms $\alpha_{L, R}^{\mu}$, the fixed point Lagrangian of the heavy mesons is expressed as \#4

$$
\begin{aligned}
\mathcal{L}_{\text {heavy }}^{*}= & -\operatorname{tr}\left[\mathcal{H}_{R} i v_{\mu} \partial^{\mu} \overline{\mathcal{H}}_{R}\right]-\operatorname{tr}\left[\mathcal{H}_{L} i v_{\mu} \partial^{\mu} \overline{\mathcal{H}}_{L}\right] \\
& +m_{0} \operatorname{tr}\left[\mathcal{H}_{R} \overline{\mathcal{H}}_{R}+\mathcal{H}_{L} \overline{\mathcal{H}}_{L}\right] \\
& +2 k \operatorname{tr}\left[\mathcal{H}_{R} \alpha_{R \mu} \gamma^{\mu} \frac{1+\gamma_{5}}{2} \overline{\mathcal{H}}_{R}+\mathcal{H}_{L} \alpha_{L \mu} \gamma^{\mu} \frac{1-\gamma_{5}}{2} \overline{\mathcal{H}}_{L}\right],
\end{aligned}
$$

where $v_{\mu}$ is the velocity of heavy meson, $m_{0}$ represents the mass generated by the interaction between heavy quark and the "pion cloud" surrounding the heavy quark, and $k$ is a real constant to be determined.

\footnotetext{
\#4 We assign the right chirality to $\mathcal{H}_{R}$, and the left chirality to $\mathcal{H}_{L}$. Then the interaction term has the right and left projection operators. Note that the insertion of $\left(1 \pm \gamma_{5}\right)$ to kinetic and mass termes does not cause any difference.
} 


\subsection{Effects of spontaneous chiral symmetry breaking}

Next we consider what happens in the broken phase of chiral symmetry. In the real world at low temperature and low density, chiral symmetry is spontaneously broken by the nonvanishing quark condensate. In the scenario of chiral-symmetry manifestation à la linear sigma model, the effect of spontaneous chiral symmetry breaking is expressed by the vacuum expectation value (VEV) of the scalar fields. In the VM, on the other hand, it is signalled by the HLS Lagrangian departing from the VM fixed point: There the gauge coupling constant $g \neq 0{ }^{\# 5}$ and we have the kinetic term of the HLS gauge bosons $\mathcal{L}_{\rho \mathrm{kin}}=-\frac{1}{2} \operatorname{tr}\left[\rho_{\mu \nu} \rho^{\mu \nu}\right]$. The derivatives in the HLS 1-forms become the covariant derivatives and then $\alpha_{L \mu}$ and $\alpha_{R \mu}$ are covariantized:

$$
\begin{gathered}
\partial_{\mu} \rightarrow D_{\mu}=\partial_{\mu}-i g \rho_{\mu}, \\
\alpha_{R \mu} \rightarrow \hat{\alpha}_{R \mu}=\alpha_{R \mu}-g \rho_{\mu}, \\
\alpha_{L \mu} \rightarrow \hat{\alpha}_{L \mu}=\alpha_{L \mu}-g \rho_{\mu} .
\end{gathered}
$$

These 1-forms transform as $\hat{\alpha}_{R(L) \mu} \rightarrow h \hat{\alpha}_{R(L) \mu} h^{\dagger}$ with $h \in\left[S U(3)_{\mathrm{V}}\right]_{\text {local }}$ as shown in Eq. (2.6) .

Although $a=1$ at the VM fixed point, generally $a \neq 1$ in the broken phase. We therefore expect to have a term of the form $\frac{1}{2}(a-1) F_{\pi}^{2} \operatorname{tr}\left[\hat{\alpha}_{L \mu} \hat{\alpha}_{R}^{\mu}\right]$. Thus the Lagrangian for the light mesons takes the following form:

$$
\begin{aligned}
\mathcal{L}_{\text {light }}=\frac{a+1}{4} & F_{\pi}^{2} \operatorname{tr}\left[\hat{\alpha}_{R \mu} \hat{\alpha}_{R}^{\mu}+\hat{\alpha}_{L \mu} \hat{\alpha}_{L}^{\mu}\right] \\
& +\frac{a-1}{2} F_{\pi}^{2} \operatorname{tr}\left[\hat{\alpha}_{R \mu} \hat{\alpha}_{L}^{\mu}\right]+\mathcal{L}_{\rho \mathrm{kin}} .
\end{aligned}
$$

By using $\hat{\alpha}_{\| \mu}$ and $\hat{\alpha}_{\perp \mu}$ given in Eq. (2.5), this Lagrangian is rewritten as

$$
\mathcal{L}_{\text {light }}=F_{\pi}^{2} \operatorname{tr}\left[\hat{\alpha}_{\perp \mu} \hat{\alpha}_{\perp}^{\mu}\right]+F_{\sigma}^{2} \operatorname{tr}\left[\hat{\alpha}_{\| \mu} \hat{\alpha}_{\|}^{\mu}\right]+\mathcal{L}_{\rho \mathrm{kin}}
$$

which is nothing but the general HLS Lagrangian.

We next consider the spontaneous breaking of chiral symmetry in the heavy-meson sector. One of the most important effects of the symmetry breaking is to generate the mass splitting between the odd parity multiplet and the even parity multiplet [1]. This effect can be represented by the Lagrangian of the form:

$$
\mathcal{L}_{\chi \mathrm{SB}}=\frac{1}{2} \Delta M \operatorname{tr}\left[\mathcal{H}_{L} \overline{\mathcal{H}}_{R}+\mathcal{H}_{R} \overline{\mathcal{H}}_{L}\right]
$$

\#5 Actually, near the chiral restoration point, the Wilsonian matching between HLS and QCD dictates [9] that (in the chiral limit) the HLS gauge coupling be proportional to the quark condensate: $g \sim\langle\bar{q} q\rangle$. 
where $\mathcal{H}_{R(L)}$ transforms under the HLS as $\mathcal{H}_{R(L)} \rightarrow \mathcal{H}_{R(L)} h^{\dagger}$. Here $\Delta M$ is the bare parameter corresponding to the mass splitting between the two multiplets. An important point of our work is that the bare $\Delta M$ can be determined by matching the EFT with QCD as we will show in section 5 . The matching actually shows that $\Delta M$ is proportional to the quark condensate:

$$
\Delta M \sim\langle\bar{q} q\rangle .
$$

\subsection{Lagrangian in parity eigenfields}

In order to compute the mass splitting between $\mathcal{M}$ and $\tilde{\mathcal{M}}$, it is convenient to go to the corresponding fields in parity eigenstate, $H$ (odd-parity) and $G$ (even-parity) as defined, e.g., in Ref. [6];

$$
\begin{aligned}
\mathcal{H}_{R} & =\frac{1}{\sqrt{2}}\left[G-i H \gamma_{5}\right], \\
\mathcal{H}_{L} & =\frac{1}{\sqrt{2}}\left[G+i H \gamma_{5}\right] .
\end{aligned}
$$

Here, the pseudoscalar meson $P$ and the vector meson $P_{\mu}^{*}$ are included in the $H$ field as

$$
H=\frac{1+v_{\mu} \gamma^{\mu}}{2}\left[i \gamma_{5} P+\gamma^{\mu} P_{\mu}^{*}\right],
$$

and the scalar meson $Q^{*}$ and the axial-vector meson $Q_{\mu}$ are in $G$ as

$$
G=\frac{1+v_{\mu} \gamma^{\mu}}{2}\left[Q^{*}-i \gamma^{\mu} \gamma_{5} Q_{\mu}\right] .
$$

In terms of the $H$ and $G$ fields, the heavy-meson Lagrangian off the VM fixed point is of the form

$$
\mathcal{L}_{\text {heavy }}=\mathcal{L}_{\text {kin }}+\mathcal{L}_{\text {int }},
$$

with

$$
\begin{aligned}
& \mathcal{L}_{\text {kin }}=\operatorname{tr}[\left.H\left(i v_{\mu} D^{\mu}-M_{H}\right) \bar{H}\right]-\operatorname{tr}\left[G\left(i v_{\mu} D^{\mu}-M_{G}\right) \bar{G}\right], \\
& \mathcal{L}_{\text {int }}=k\left[\operatorname{tr}\left[H \gamma_{\mu} \gamma_{5} \hat{\alpha}_{\perp}^{\mu} \bar{H}\right]-\operatorname{tr}\left[H v_{\mu} \hat{\alpha}_{\|}^{\mu} \bar{H}\right]\right. \\
& \quad+\operatorname{tr}\left[G \gamma_{\mu} \gamma_{5} \hat{\alpha}_{\perp}^{\mu} \bar{G}\right]+\operatorname{tr}\left[G v_{\mu} \hat{\alpha}_{\|}^{\mu} \bar{G}\right] \\
& \quad-i \operatorname{tr}\left[G \hat{\alpha}_{\perp \mu} \gamma^{\mu} \gamma_{5} \bar{H}\right]+i \operatorname{tr}\left[H \hat{\alpha}_{\perp \mu} \gamma^{\mu} \gamma_{5} \bar{G}\right] \\
&\left.-i \operatorname{tr}\left[G \hat{\alpha}_{\| \mu} \gamma^{\mu} \bar{H}\right]+i \operatorname{tr}\left[H \hat{\alpha}_{\| \mu} \gamma^{\mu} \bar{G}\right]\right]
\end{aligned}
$$

where the covariant derivatives acting on $\bar{H}$ and $\bar{G}$ are defined as

$$
D_{\mu} \bar{H}=\left(\partial_{\mu}-i g \rho_{\mu}\right) \bar{H}, \quad D_{\mu} \bar{G}=\left(\partial_{\mu}-i g \rho_{\mu}\right) \bar{G} .
$$


In the above expression, $M_{H}$ and $M_{G}$ denote the masses of the parity-odd multiplet $H$ and the parity-even multiplet $G$, respectively. They are related to $m_{0}$ and $\Delta M$ as

$$
\begin{aligned}
& M_{H}=-m_{0}-\frac{1}{2} \Delta M, \\
& M_{G}=-m_{0}+\frac{1}{2} \Delta M .
\end{aligned}
$$

The mass splitting between $G$ and $H$ is therefore given by

$$
M_{G}-M_{H}=\Delta M
$$

\section{MATCHING TO THE OPERATOR PRODUCT EXPANSION}

The bare parameter $\Delta M_{\text {bare }}$ which carries information on QCD should be determined by matching the EFT correltators to QCD ones. We are concerned with the pseudoscalar correlator $G_{P}$ and the scalar correlator $G_{S}$. In the EFT sector, the correlators at the matching scale are of the form $\# 6$

$$
\begin{aligned}
& G_{P}\left(Q^{2}\right)=\frac{F_{D}^{2} M_{D}^{4}}{M_{D}^{2}+Q^{2}}, \\
& G_{S}\left(Q^{2}\right)=\frac{F_{\tilde{D}}^{2} M_{\tilde{D}}^{4}}{M_{\tilde{D}}^{2}+Q^{2}},
\end{aligned}
$$

where $F_{D}\left(F_{\tilde{D}}\right)$ denotes the $D$-meson ( $\tilde{D}$-meson) decay constant and the space-like momentum $Q^{2}=\left(M_{D}+\Lambda_{M}\right)^{2}$ with $\Lambda_{M}$ being the matching scale. We note that the heavy quark limit $M_{D} \rightarrow \infty$ should be taken with $\Lambda_{M}$ kept fixed since $\Lambda_{M}$ must be smaller than the chiral symmetry breaking scale characterized by $\Lambda_{\chi} \sim 4 \pi f_{\pi}$. Then, $Q^{2}$ should be regarded as $Q^{2} \simeq$ $M_{D}^{2}$ in the present framework based on the chiral and heavy quark symmetries. If we ignore the difference between $F_{D}$ and $F_{\tilde{D}}$ which can be justified by the QCD sum rule analysis [20], then we get

$$
\Delta_{S P}\left(Q^{2}\right) \equiv G_{S}\left(Q^{2}\right)-G_{P}\left(Q^{2}\right) \simeq \frac{3 F_{D}^{2} M_{D}^{3}}{M_{D}^{2}+Q^{2}} \Delta M_{D}
$$

In the QCD sector, the correlators $G_{S}$ and $G_{P}$ are given by the operator product expansion (OPE) as [21]

$$
G_{S}\left(Q^{2}\right)=\left.G\left(Q^{2}\right)\right|_{\text {pert }}
$$

\#6 Here and in the rest of the paper, the heavy meson is denoted $D$ with the open charm heavy meson in mind. However the arguments (except for the numerical values) are generic for all heavy mesons $\mathcal{M}$. 


$$
\begin{aligned}
& +\frac{m_{H}^{2}}{m_{H}^{2}+Q^{2}}\left[-m_{H}\langle\bar{q} q\rangle+\frac{\alpha_{s}}{12 \pi}\left\langle G^{\mu \nu} G_{\mu \nu}\right\rangle\right], \\
G_{P}\left(Q^{2}\right) & =\left.G\left(Q^{2}\right)\right|_{\text {pert }} \\
& +\frac{m_{H}^{2}}{m_{H}^{2}+Q^{2}}\left[m_{H}\langle\bar{q} q\rangle+\frac{\alpha_{s}}{12 \pi}\left\langle G^{\mu \nu} G_{\mu \nu}\right\rangle\right],
\end{aligned}
$$

where $m_{H}$ is the heavy-quark mass. To the accuracy we are aiming at, the OPE can be truncated at $\mathcal{O}\left(1 / Q^{2}\right)$. The explicit expression for the perturbative contribution $\left.G\left(Q^{2}\right)\right|_{\text {pert }}$ is available in the literature but we do not need it since it drops out in the difference. From these correlators, the $\Delta_{S P}$ becomes

$$
\Delta_{S P}\left(Q^{2}\right)=-\frac{2 m_{H}^{3}}{m_{H}^{2}+Q^{2}}\langle\bar{q} q\rangle .
$$

Equating Eq. (3.2) to Eq. (3.4) and neglecting the difference $\left(m_{H}-M_{D}\right)$, we obtain the following matching condition:

$$
3 F_{D}^{2} \Delta M \simeq-2\langle\bar{q} q\rangle
$$

Thus at the matching scale, the splitting is

$$
\Delta M_{\text {bare }} \simeq-\frac{2}{3} \frac{\langle\bar{q} q\rangle}{F_{D}^{2}} .
$$

As announced, the bare splitting is indeed proportional to the light-quark condensate. The quantum corrections do not change the dependence on the quark condensate [see section 4 .

\section{QUANTUM CORRECTIONS AND RGE}

Given the bare Lagrangian whose parameters are fixed at the matching scale $\Lambda_{M}$, the next step is to decimate the theory à la Wilson to the scale at which $\Delta M$ is measured. This amounts to calculating quantum corrections to the mass difference $\Delta M$ in the framework of the present EFT.

This calculation turns out to be surprisingly simple for $a \approx 1$. If one sets $a=1$ which is the approximation we are adopting here, $\alpha_{L}$ does not mix with $\alpha_{R}$ in the light sector, and then $\alpha_{L}$ couples to only $\mathcal{H}_{L}$ and $\alpha_{R}$ to only $\mathcal{H}_{R}$. As a result $\mathcal{H}_{L(R)}$ cannot connect to $\mathcal{H}_{R(L)}$ by the exchange of $\alpha_{L}$ or $\alpha_{R}$. Only the $\rho$-loop links between the fields with different chiralities as shown in Fig. 1. We have verified this approximation to be reliable since corrections to the result with $a=1$ come only at higher loop orders [see the next paragraph]. The diagram shown in Fig. 1 1 contributes to the two-point function as

$$
\left.\Pi_{L R}\right|_{\text {div }}=-\frac{1}{2} \Delta M \mathcal{C}_{2}\left(N_{f}\right) \frac{g^{2}}{2 \pi^{2}}\left(1-2 k-k^{2}\right) \ln \Lambda,
$$




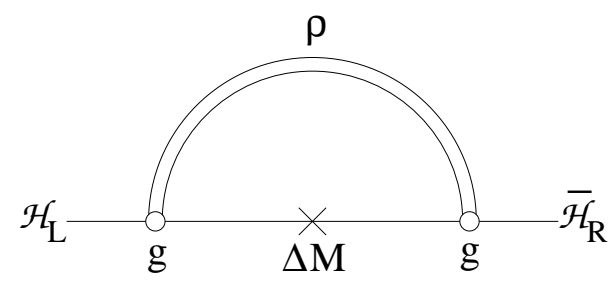

FIG. 1: Diagram contributing to the mass difference.

where $\mathcal{C}_{2}\left(N_{f}\right)$ is the second Casimir defined by $\left(T_{a}\right)_{i j}\left(T_{a}\right)_{j l}=\mathcal{C}_{2}\left(N_{f}\right) \delta_{i l}$ with $i, j$ and $l$ denoting the flavor indices of the light quarks. This divergence is renormalized by the bare contribution of the form $\Pi_{L R \text {, bare }}=\frac{1}{2} \Delta M_{\text {bare }}$. Thus the renormalization-group equation (RGE) takes the form

$$
\mu \frac{d \Delta M}{d \mu}=\mathcal{C}_{2}\left(N_{f}\right) \frac{g^{2}}{2 \pi^{2}}\left(1-2 k-k^{2}\right) \Delta M
$$

For an approximate estimate that we are interested in at this point, it seems reasonable to ignore the scale dependence in $g$ and $k$. Then the solution is simple:

$$
\Delta M=\Delta M_{\text {bare }} \times C_{\text {quantum }}
$$

where we define $C_{\text {quantum }}$ by

$$
C_{\text {quantum }}=\exp \left[-\mathcal{C}_{2}\left(N_{f}\right) \frac{g^{2}}{2 \pi^{2}}\left(1-2 k-k^{2}\right) \ln \frac{\Lambda}{\mu}\right]
$$

This shows unequivocally that the mass splitting is dictated by the "bare" splitting $\Delta M_{\text {bare }}$ proportional to $\langle\bar{q} q\rangle$ corrected by the quantum effect $C_{\text {quantum }}$.

Next we lift the condition $a=1$ made in the above analysis. For this purpose, we compute the quantum effects to the masses of $0^{-}(P)$ and $0^{+}\left(Q^{*}\right) D$-mesons by calculating the one-loop corrections to the two-point functions of $P$ and $Q^{*}$ denoted by $\Pi_{P P}$ and $\Pi_{Q^{*} Q^{*}}$ [for the explicit calculation, see Appendix $\mathrm{A}$. We find that amazingly, the resultant form of the quantum correction exactly agrees with the previous one which was obtained by taking $a=1$. To arrive at this result, it is essential that $P$ (or $P_{\mu}^{*}$ ) be the chiral partner of $Q^{*}$ (or $Q_{\mu}$ ) as follows: The loop diagrams shown in Fig. 2 and Fig. 3 in Appendix $\mathrm{A}$ have power and logarithmic divergences. However all the divergences of the diagrams with pion loop are exactly canceled among themselves since the internal (or external) particles are chiral partners. In a similar way, the exact cancellation takes place in the diagrams with $\sigma$ loop. Finally, the logarithmic divergence from the $\rho$ loop does contribute to the mass difference. This shows that the effect of spontaneous chiral symmetry breaking introduced as the deviation of $a$ from 1 does not get transferred to the heavy sector. Thus even in the case of $a \neq 1$, the bare mass splitting is 
enhanced by only the vector meson loop, with the pions not figuring in the quantum corrections at least at one-loop order. Solving the RGE (A.11), which is exactly same as Eq. (4.2), we obtain exactly the same mass splitting as the one given in Eq. (4.3).

\section{MASS SPLITTING}

In this section we make a numerical estimation of the mass splitting for the chiral doublers in the open charm system. (Here $D$ denotes the open charm meson.) Since we are considering the chiral limit, strictly speaking, a precise comparison with experiments is not feasible particularly if the light quark is strange, so what we obtain should be considered as semi-quantitative at best. This caveat should be kept in mind in what follows.

Determining the bare mass splitting from the matching condition (3.6) requires the quark condensate at that scale and the $D$-meson decay constant $F_{D}$. For the quark condensate, we shall use the so-called "standard value" [22] $\langle\bar{q} q\rangle=-(225 \pm 25 \mathrm{MeV})^{3}$ at $1 \mathrm{GeV}$. Extrapolated to the scale $\Lambda_{M}=1.1 \mathrm{GeV}$ we shall adopt here, this gives

$$
\langle\bar{q} q\rangle_{\Lambda_{M}}=-(228 \pm 25 \mathrm{MeV})^{3}
$$

Unfortunately this value is not firmly established, there being no consensus on it. The values found in the literature vary widely, even by a factor of $\sim 2$, some higher [23] and some lower 24]. (See Appendix B for more on this matter.) We shall therefore take the standard value as a median $\# 7$. As for the $D$-meson decay constant, we take as a typical value $F_{D}=$ $0.205 \pm 0.020 \mathrm{GeV}$ obtained from the QCD sum rule analysis [20]. Plugging the above input values into Eq. (3.6) we obtain

$$
\Delta M_{\text {bare }} \simeq 0.19 \mathrm{GeV} \text {. }
$$

By taking $\mu=m_{\rho}=771 \mathrm{MeV}, \Lambda=\Lambda_{M}=1.1 \mathrm{GeV}, g=g\left(m_{\rho}\right)=6.27$ determined via the Wilsonian matching for $\left(\Lambda_{M}, \Lambda_{\mathrm{QCD}}\right)=(1.1,0.4) \mathrm{GeV}$ in Ref. [9] and $k \simeq 0.59$ extracted from the $D^{*} \rightarrow D \pi$ decay [see section 6.1] in Eq. (4.4), we find for $N_{F}=3$

$$
C_{\text {quantum }}=1.6
$$

\#7 It was shown in Ref. 24] that there is a strong $N_{F}$ dependence on the quark condensate and the value of the quark condensate for QCD with three massless quarks is smaller than the value used in estimating the value of the mass splitting in section 5. In the present analysis, we extract the value of the coupling constant $k$ from the experiment. To be consistent, we need to use the quark condensate together with other parameters involved determined at the same scale from experimental and/or lattice data. This corresponds to the standard value of the condensate we are using here. 
This is a sizable quantum correction involving only the vector meson. If one takes into account the uncertainties involved in the condensate and the decay constant, the quantum-corrected splitting $\Delta M$ comes out to be

$$
\Delta M \approx 0.31 \pm 0.12 \mathrm{GeV}
$$

Despite the uncertainty involved, (5.4) is a pleasing result. It shows that the splitting is indeed of the size of the constituent quark mass of a chiral quark $\Sigma \sim m_{p} / 3 \sim 310 \mathrm{MeV}$ and is directly proportional to the quark condensate.

We should stress however several caveats associated with this result. Apart from the sensitivity to the quark condensate, if one naively plugs in the matching scale $\Lambda_{M}$ into the RGE solution, one finds the splitting is not insensitive as it should be to the scale change. This is neither surprising nor too disturbing since our RGE solution is obtained with the scale dependence in both $g$ and $k$ ignored. In order to eliminate this dependence on the matching scale, it will be necessary to solve the RGE with the full scale dependence taken into account - which is at the moment beyond our scope here. The best we can do within the scheme adopted is to pick the optimal $\Lambda_{M}$ determined phenomenologically from elsewhere [9] and this is what we have done above.

\section{HADRONIC DECAY MODES}

In this section we turn to the hadronic decay processes of the $\tilde{D}$ mesons and make predictions of our scenario based on the vector manifestation (VM) of chiral symmetry. Here we adopt the notations $D_{u, d}$ and $\tilde{D}_{u, d}$ for the heavy ground-state mesons and heavy excited mesons composed of $c \bar{u}$ and $c \bar{d}$, and $D_{s}$ and $\tilde{D}_{s}$ for those composed of $c \bar{s}$. The spin-parity quantum numbers will be explicitly written as $D_{u, d}\left(0^{-}\right)$. For the heavy vector meson, we follow the notation adopted by the Particle Data Group (PDG) [25] and write $D_{u, d}^{*}\left(1^{-}\right)$and $D_{s}^{*}\left(1^{-}\right)$. Unless otherwise noted, the masses of the ground-state heavy mesons will be denoted as $M_{D}$ and those of the excited states as $M_{\tilde{D}}$.

\section{1. $D^{*} \rightarrow D+\pi$}

Before studying the decay processes of the excited heavy mesons, we first calculate the decay width of $D_{u, d}^{*} \rightarrow D_{u, d}+\pi$ so as to determine the coupling constant $k$. The decay widths of the $\pi^{0}$ and the $\pi^{ \pm}$modes are given by

$$
\Gamma\left(D_{u, d}^{*}\left(1^{-}\right) \rightarrow D_{u, d}\left(0^{-}\right)+\pi^{0}\right)=\frac{\bar{p}_{\pi}^{3}}{24 \pi M_{D^{*}}^{2}}\left(M_{Q} \frac{k}{F_{\pi}}\right)^{2},
$$




$$
\Gamma\left(D_{u, d}^{*}\left(1^{-}\right) \rightarrow D_{u, d}\left(0^{-}\right)+\pi^{ \pm}\right)=\frac{\bar{p}_{\pi}^{3}}{12 \pi M_{D^{*}}^{2}}\left(M_{Q} \frac{k}{F_{\pi}}\right)^{2},
$$

where $\bar{p}_{\pi} \equiv\left|\vec{p}_{\pi}\right|$ denotes the three-momentum of the pion in the rest frame of the decaying particle $D_{u, d}^{*}\left(1^{-}\right)$, and $M_{Q}$ the "heavy quark mass" introduced for correctly normalizing the heavy meson field. In the present analysis we use the following reduced mass for definiteness:

$$
M_{Q} \equiv \frac{1}{4}\left(M_{D\left(0^{-}\right)}+3 M_{D^{*}\left(1^{-}\right)}\right)=1974 \mathrm{MeV} .
$$

The total width is not determined for $D_{u}^{*}\left(1^{-}\right)$, although the branching fractions for both the $\pi^{0}$ and the $\pi^{+}$decay modes are known experimentally. For $D_{d}^{*}\left(1^{-}\right)$meson, on the other hand, the total width is also determined. Using the values listed in PDG table [25], the partial decay widths are estimated to be

$$
\begin{aligned}
& \Gamma\left(D_{d}^{*}\left(1^{-}\right) \rightarrow D_{d}\left(0^{-}\right)+\pi^{0}\right)=29.5 \pm 6.8 \mathrm{keV}, \\
& \Gamma\left(D_{d}^{*}\left(1^{-}\right) \rightarrow D_{u}\left(0^{-}\right)+\pi^{+}\right)=65 \pm 15 \mathrm{keV} .
\end{aligned}
$$

Here the $\pi^{0}$ mode will be used as an input to fix $k$. From the experimental masses $M_{D_{u}^{*}\left(1^{-}\right)}=$ 2010.1 MeV, $M_{D_{d}\left(0^{-}\right)}=1869.4 \mathrm{MeV}$ and $M_{\pi^{0}}=134.9766 \mathrm{MeV}$ together with the value of the pion decay constant $F_{\pi}=92.42 \pm 0.26 \mathrm{MeV}$, we obtain

$$
k=0.59 \pm 0.07 .
$$

Note that the error is mainly from that of the $D_{d}^{*}\left(1^{-}\right) \rightarrow D_{d}\left(0^{-}\right)+\pi^{0}$ decay width.

In the following analysis, we shall use the central value of $k$ to make predictions for the decay widths of $\tilde{D}$ mesons. Each prediction includes at least about $20 \%$ error from the value of $k$. For the masses of excited $D$ mesons, we use $M_{\tilde{D_{s}\left(0^{+}\right)}}=2317 \mathrm{MeV}$ determined by BaBar 3], $M_{\tilde{D}_{s}\left(1^{+}\right)}=2460 \mathrm{MeV}$ by CLEO [4] and $\left(M_{\tilde{D}_{u, d}\left(0^{+}\right)}, M_{\tilde{D}_{u, d}\left(1^{+}\right)}\right)=(2308,2427) \mathrm{MeV}$ by Belle [5]. Table \summarizes the input parameters used in the present analysis.

\section{2. $\quad \tilde{D} \rightarrow D+\pi$}

For the systems of $c \bar{u}$ and $c \bar{d}$, the following decay processes of the $\tilde{D}_{u, d}$ meson into the $D_{u, d}$ meson and one pion are allowed by the spin and parity:

$$
\tilde{D}_{u, d}\left(0^{+}\right) \rightarrow D_{u, d}\left(0^{-}\right)+\pi \quad \tilde{D}_{u, d}\left(1^{+}\right) \rightarrow D_{u, d}^{*}\left(1^{-}\right)+\pi .
$$

Their partial decay widths are given by

$$
\begin{aligned}
\Gamma\left(\tilde{D}_{u, d} \rightarrow D_{u, d}+\pi^{ \pm}\right) & =\frac{\bar{p}_{\pi}}{4 \pi}\left(\frac{k}{F_{\pi}} \frac{M_{Q}}{M_{\tilde{D}}} E_{\pi}\right)^{2}, \\
\Gamma\left(\tilde{D}_{u, d} \rightarrow D_{u, d}+\pi^{0}\right) & =\frac{\bar{p}_{\pi}}{8 \pi}\left(\frac{k}{F_{\pi}} \frac{M_{Q}}{M_{\tilde{D}}} E_{\pi}\right)^{2},
\end{aligned}
$$




\begin{tabular}{ccccc}
\hline$D_{u, d}$ meson masses & $M_{\tilde{D}_{u, d}\left(1^{+}\right)}$ & $M_{\tilde{D}_{u, d}\left(0^{+}\right)}$ & $M_{D_{u, d}^{*}\left(1^{-}\right)}$ & $M_{D_{u, d}\left(0^{-}\right)}$ \\
$(\mathrm{MeV})$ & 2427 & 2308 & 2010 & 1865 \\
\hline$D_{s}$ meson masses & $M_{\tilde{D}_{s}\left(1^{+}\right)}$ & $M_{\tilde{D}_{s}\left(0^{+}\right)}$ & $M_{D_{s}^{*}\left(1^{-}\right)}$ & $M_{D_{s}\left(0^{-}\right)}$ \\
$(\mathrm{MeV})$ & 2460 & 2317 & 2112 & 1969 \\
\hline Light meson masses & $M_{\pi}$ & $M_{\rho}$ & $M_{\eta}$ & $M_{\phi}$ \\
$(\mathrm{MeV})$ & 138.039 & 771.1 & 547.30 & 1019.456 \\
\hline$\pi^{0}-\eta$ mixing & $A_{11}$ & $A_{21}$ & $\Pi_{\pi^{0} \eta}(\mathrm{MeV})^{2}$ & $K_{\pi^{0} \eta}$ \\
& 0.71 & -0.52 & $-4.25 \times 10^{3}$ & $-1.06 \times 10^{-2}$ \\
\hline$\phi-\rho$ mixing & $\Gamma_{\phi \rightarrow \pi^{+} \pi^{-}}(\mathrm{MeV})$ & $\Gamma_{\rho \rightarrow \pi^{+} \pi^{-}}(\mathrm{MeV})$ & & \\
\multicolumn{5}{c}{} \\
\hline
\end{tabular}

TABLE I: The values of input parameters. We use the values of $M_{\tilde{D}_{s}\left(0^{+}\right)}$[3], $M_{\tilde{D}_{s}\left(1^{+}\right)}$[4] and $M_{\tilde{D}_{u, d}\left(0^{+}, 1^{+}\right)}[5]$. The $D$ mesons in the ground state, light mesons and decay widths $\Gamma(\phi, \rho)$ are the values listed by the PDG table [25]. As for the parameters associated with the $\pi^{0}-\eta$ mixing, we use the values given in Refs. [26, 27].

where $E_{\pi}$ is the energy of the pion, and the reduced mass $M_{Q}$ is defined in Eq. (6.2). With the input parameters given in Table \ these decay widths come out to be

$$
\begin{aligned}
& \Gamma\left(\tilde{D}_{u, d}\left(0^{+}\right) \rightarrow D_{u, d}\left(0^{-}\right)+\pi^{0}\right)=73.7 \mathrm{MeV}, \\
& \Gamma\left(\tilde{D}_{u, d}\left(0^{+}\right) \rightarrow D_{u, d}\left(0^{-}\right)+\pi^{ \pm}\right)=147 \mathrm{MeV}, \\
& \Gamma\left(\tilde{D}_{u, d}\left(1^{+}\right) \rightarrow D_{u, d}^{*}\left(1^{-}\right)+\pi^{0}\right)=57.2 \mathrm{MeV}, \\
& \Gamma\left(\tilde{D}_{u, d}\left(1^{+}\right) \rightarrow D_{u, d}^{*}\left(1^{-}\right)+\pi^{ \pm}\right)=114 \mathrm{MeV},
\end{aligned}
$$

For the system of $c \bar{s}$ there are two decay processes of the $\tilde{D}_{s}$ meson into the $D_{s}$ meson and one pion:

$$
\tilde{D}_{s}\left(0^{+}\right) \rightarrow D_{s}\left(0^{-}\right)+\pi^{0} \quad \tilde{D}_{s}\left(1^{+}\right) \rightarrow D_{s}^{*}\left(1^{-}\right)+\pi^{0} .
$$

These processes violate the isospin invariance, and hence are suppressed. In the present analysis we assume as in Ref. [28] that the isospin violation occurs dominantly through the $\pi^{0}-\eta$ mixing. In other words, we assume that the $\tilde{D}_{s}$ meson decays into the $D_{s}$ meson and the virtual $\eta$ meson which mixes with the $\pi^{0}$ through the $\pi^{0}-\eta$ mixing. Then, the decay width is given by

$$
\Gamma\left(\tilde{D}_{s} \rightarrow D_{s}+\pi^{0}\right)=\frac{\bar{p}_{\pi}}{2 \pi}\left(\frac{k}{F_{\pi}} \frac{M_{Q}}{M_{\tilde{D}}} E_{\pi} \Delta_{\pi^{0} \eta}\right)^{2},
$$

where $\Delta_{\pi^{0} \eta}$ denotes the $\pi^{0}-\eta$ mixing and takes the following form [26, 27]:

$$
\Delta_{\pi^{0} \eta}=-\frac{A_{11} A_{21}}{M_{\eta}^{2}-M_{\pi^{0}}^{2}}\left(\Pi_{\pi^{0} \eta}-K_{\pi^{0} \eta} M_{\pi^{0}}^{2}\right)
$$


with $\Pi_{\pi^{0} \eta}$ and $K_{\pi^{0} \eta}$ being the mass-type and kinetic-type $\pi^{0}-\eta$ mixing, respectively. $A_{11}$ and $A_{21}$ are the components of the $\eta-\eta^{\prime}$ mixing matrix in the two-mixing-angle scheme [26]. By

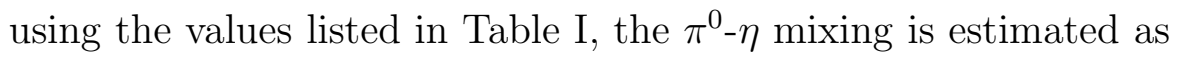

$$
\Delta_{\pi^{0} \eta}=-5.32 \times 10^{-3}
$$

From this value, the decay widths are predicted as

$$
\begin{aligned}
& \Gamma\left(\tilde{D}_{s}\left(0^{+}\right) \rightarrow D_{s}\left(0^{-}\right)+\pi^{0}\right)=4.17 \mathrm{keV}, \\
& \Gamma\left(\tilde{D}_{s}\left(1^{+}\right) \rightarrow D_{s}^{*}\left(1^{-}\right)+\pi^{0}\right)=3.75 \mathrm{keV} .
\end{aligned}
$$

6.3. $\tilde{D}\left(1^{+}\right) \rightarrow \tilde{D}\left(0^{+}\right)+\pi$

With the masses of $\tilde{D}_{u, d}\left(1^{+}\right)$and $\tilde{D}_{u, d}\left(0^{+}\right)$listed in Table 【 the intra-multiplet decay $\tilde{D}_{u, d}\left(1^{+}\right) \rightarrow \tilde{D}_{u, d}\left(0^{+}\right)+\pi$ is not allowed kinematically. Since the experimental errors for the masses are large ${ }^{\# 8}$, this decay mode may still turn out to be possible. To show how large the possible decay width is, we use $M_{\tilde{D}_{u, d}\left(0^{+}\right)}=2272 \mathrm{MeV}$ and $M_{\tilde{D}_{u, d}\left(1^{+}\right)}=2464 \mathrm{MeV}$ together with the formulas

$$
\begin{aligned}
& \Gamma\left(\tilde{D}_{u, d}\left(1^{+}\right) \rightarrow \tilde{D}_{u, d}\left(0^{+}\right)+\pi^{0}\right)=\frac{\bar{p}_{\pi}^{3}}{24 \pi}\left(\frac{M_{Q}}{M_{\tilde{D}\left(1^{+}\right)}} \frac{k}{F_{\pi}}\right)^{2}, \\
& \Gamma\left(\tilde{D}_{u, d}\left(1^{+}\right) \rightarrow \tilde{D}_{u, d}\left(0^{+}\right)+\pi^{+}\right)=\frac{\bar{p}_{\pi}^{3}}{12 \pi}\left(\frac{M_{Q}}{M_{\tilde{D}\left(1^{+}\right)}} \frac{k}{F_{\pi}}\right)^{2} .
\end{aligned}
$$

The resultant decay widths are given by

$$
\begin{aligned}
& \Gamma\left(\tilde{D}_{u, d}\left(1^{+}\right) \rightarrow \tilde{D}_{u, d}\left(0^{+}\right)+\pi^{0}\right)=0.729 \mathrm{MeV} \\
& \Gamma\left(\tilde{D}_{u, d}\left(1^{+}\right) \rightarrow \tilde{D}_{u, d}\left(0^{+}\right)+\pi^{+}\right)=1.46 \mathrm{MeV}
\end{aligned}
$$

They are smaller by the order of $10^{-2}$ than other one-pion modes [see Table II]. This is caused by the suppression from the phase space.

With the present input values of $\tilde{D}$ masses, the process $\tilde{D}_{s}\left(1^{+}\right) \rightarrow \tilde{D}_{s}\left(0^{+}\right)+\pi^{0}$ is kinematically allowed. Similarly to the $\tilde{D}_{s} \rightarrow D_{s}+\pi^{0}$ decay, we assume that this decay is dominated by the process through the $\pi^{0}-\eta$ mixing. Then, the decay width is given by

$$
\begin{aligned}
\Gamma\left(\tilde{D}_{s}\left(1^{+}\right) \rightarrow \tilde{D}_{s}\left(0^{+}\right)+\pi^{0}\right) & =\frac{\bar{p}_{\pi}^{3}}{6 \pi}\left(\frac{k}{F_{\pi}} \frac{M_{Q}}{M_{\tilde{D}\left(1^{+}\right)}} \Delta_{\pi^{0} \eta}\right)^{2} \\
& =1.87 \times 10^{-3} \mathrm{keV}
\end{aligned}
$$

This is very tiny due to the isospin violation and the phase-space suppression.

\#8 The Belle collaboration 299, 30, 31] gives $M_{\tilde{D}_{u, d}\left(1^{+}\right)}=2427 \pm 26 \pm 20 \pm 17 \mathrm{MeV}$ and $M_{\tilde{D}_{u, d}\left(0^{+}\right)}=2308 \pm 17 \pm$ $15 \pm 28 \mathrm{MeV}$. 


\section{4. $\quad \tilde{D} \rightarrow D+2 \pi$}

There are several processes such as $\tilde{D} \rightarrow D+\pi^{ \pm} \pi^{\mp}$ to which the light scalar mesons could give important contributions. In models based on the standard scenario of the chiral symmetry restoration in the light quark sector, the scalar-meson coupling to the heavy-quark system is related to the pion coupling, enabling one to compute the decay width. In our model based on the VM of the chiral symmetry restoration, on the other hand, it is the coupling constant of the vector meson to the heavy system that is related to the pion coupling constant: Here coupling of the scalar meson is not directly connected, at least in the present framework which contains no explicit scalar fields $\# 9$, to do that of the pion. So, while we cannot make firm predictions to processes for which scalar mesons might contribute, we can make definite predictions on certain decay widths for which scalar mesons do not figure. If one ignores isospin violation, the two-pion decay processes $\tilde{D}_{u, d} \rightarrow D_{u, d}+\pi^{ \pm} \pi^{0}$ receive no contributions from scalar mesons. We give predictions for these processes below. As for the two-pion decay modes of the $\tilde{D}_{s}$ meson, the scalar mesons could give a contribution. To have an idea, we shall also compute the vector-meson contribution to this process.

First, consider $\tilde{D}_{u, d}\left(0^{+}\right) \rightarrow D_{u, d}^{*}\left(1^{-}\right)+\pi^{ \pm} \pi^{0}$. In this process, there are two contributions:

$$
\begin{aligned}
& \tilde{D}_{u, d}\left(0^{+}\right) \rightarrow D_{u, d}^{*}\left(1^{-}\right)+\pi^{ \pm} \pi^{0} \\
& \left.D_{u, d}^{*}\left(1^{-}\right)+\left(\rho^{ \pm} \rightarrow \pi^{ \pm} \pi^{0}\right) \quad \text { ( } \rho \text {-mediation }\right) .
\end{aligned}
$$

The decay width is given by

$$
\begin{aligned}
\Gamma\left(\tilde{D}_{u, d}\left(0^{+}\right) \rightarrow D_{u, d}^{*}\left(1^{-}\right)+\pi^{ \pm} \pi^{0}\right) \\
=\frac{M_{Q}^{2}}{64(2 \pi)^{3} M_{\tilde{D}}^{3}} \frac{k^{2}}{F_{\pi}^{4}} \int d m_{D \pi}^{2} \int d m_{\pi \pi}^{2}\left|F_{\tilde{D} D}\right|^{2} \\
\quad \times\left[m_{\pi \pi}^{2}-4 M_{\pi}^{2}+\frac{1}{4 M_{D}^{2}}\left(m_{\pi \pi}^{2}-M_{\tilde{D}}^{2}-M_{D}^{2}-2 M_{\pi}^{2}+2 m_{D \pi}^{2}\right)^{2}\right],
\end{aligned}
$$

with $m_{D \pi}^{2}=\left(p_{D}+p_{\pi}\right)^{2}$ and $m_{\pi \pi}^{2}=\left(p_{1 \pi}+p_{2 \pi}\right)^{2}$. The form factor $F_{\tilde{D} D}$ is taken to be of the form

$$
F_{\tilde{D} D}=1+\frac{M_{\rho}^{2}}{m_{\pi \pi}^{2}-M_{\rho}^{2}} .
$$

The first term of the form factor comes from the direct contribution and the second from the $\rho$-mediation. Here we have neglected the $\rho$ meson width in the propagator, since the maximum

\#9 Scalar excitations can of course be generated at high loop level to assure unitarity or with the account of QCD trace anomaly but we shall not attempt this extension in this paper. 
value of $m_{\pi \pi}$ is about $300 \mathrm{MeV}$ with the input values listed in Table【. We can see that the form factor $F_{\tilde{D} D}$ vanishes in the limit of $m_{\pi \pi} \rightarrow 0$, which is a consequence of chiral symmetry ${ }^{\# 10}$. We note that $\left.m_{\pi \pi}\right|_{\max } \simeq 300 \mathrm{MeV}$ makes this decay width strongly suppressed due to the large cancellation between the direct and $\rho$-mediated contributions. Furthermore, since $300 \mathrm{MeV}$ is close to the two-pion threshold, additional suppression comes from the phase space. Due to these two types of suppressions the predicted decay width is predicted to be very small, of the order of $10^{-2} \mathrm{keV}$. \#11

Next we consider the process $\tilde{D}_{u, d}\left(1^{+}\right) \rightarrow D_{u, d}^{*}\left(1^{-}\right)+\pi \pi$. Again there are two contributions, direct and a $\rho$-mediated:

$$
\begin{aligned}
& \tilde{D}_{u, d}\left(1^{+}\right) \rightarrow D_{u, d}^{*}\left(1^{-}\right)+\pi^{ \pm} \pi^{0} \quad \text { (direct) } \\
& D_{u, d}^{*}\left(1^{-}\right)+\left(\rho^{ \pm} \rightarrow \pi^{ \pm} \pi^{0}\right) \quad(\rho \text {-mediation })
\end{aligned}
$$

The resultant decay width is given by

$$
\begin{aligned}
& \Gamma\left(\tilde{D}_{u, d}\left(1^{+}\right) \rightarrow D_{u, d}^{*}\left(1^{-}\right)+\pi^{ \pm} \pi^{0}\right) \\
& =\frac{M_{Q}^{2}}{96(2 \pi)^{3} M_{\tilde{D}}^{3}} \frac{k^{2}}{F_{\pi}^{4}} \int d m_{D \pi}^{2} \int d m_{\pi \pi}^{2}\left|F_{\tilde{D} D}\right|^{2} \\
& \quad \times\left[m_{\pi \pi}^{2}-4 M_{\pi}^{2}+\frac{1}{4 M_{\tilde{D}}^{2}}\left(m_{\pi \pi}^{2}-M_{\tilde{D}}^{2}-M_{D}^{2}-2 M_{\pi}^{2}+2 m_{D \pi}^{2}\right)^{2}\right] .
\end{aligned}
$$

Similarly to $\Gamma\left(\tilde{D}_{u, d}\left(0^{+}\right) \rightarrow D_{u, d}^{*}\left(1^{-}\right)+\pi^{ \pm} \pi^{0}\right)$, the width is again suppressed due to the large cancellation between the direct and $\rho$-mediated contributions. The suppression from the phase space, on the other hand, is not so large since $\left.m_{\pi \pi}\right|_{\max } \simeq 420 \mathrm{MeV}$ is not so close to the two-pion threshold. The resulting decay width is

$$
\Gamma\left(\tilde{D}_{u, d}\left(1^{+}\right) \rightarrow D_{u, d}^{*}\left(1^{-}\right)+\pi^{ \pm} \pi^{0}\right)=11.8 \mathrm{keV}
$$

The decay width of the process

$$
\begin{aligned}
& \tilde{D}_{u, d}\left(1^{+}\right) \rightarrow D_{u, d}\left(0^{-}\right)+\pi^{ \pm} \pi^{0} \quad \text { (direct) } \\
& D_{u, d}\left(0^{-}\right)+\left(\rho^{ \pm} \rightarrow \pi^{ \pm} \pi^{0}\right) \quad(\rho \text {-mediation })
\end{aligned}
$$

\#10 It should be stressed that this cancellation occurs because the vector meson is included consistently with chiral symmetry, and that it is not a specific feature of the VM. The chiral symmetry restoration based on the VM implies that the coupling constant of the vector meson to the heavy system is equal to that of the pion.

\#11 Note that the prediction on the decay width is very sensitive to the precise value of the mass of $\tilde{D}\left(0^{+}\right)$meson. 
is given by

$$
\begin{aligned}
\Gamma\left(\tilde{D}_{u, d}\left(1^{+}\right) \rightarrow D_{u, d}\left(0^{-}\right)+\pi^{ \pm} \pi^{0}\right) \\
=\frac{M_{Q}^{2}}{192(2 \pi)^{3} M_{\tilde{D}}^{3}} \frac{k^{2}}{F_{\pi}^{4}} \int d m_{D \pi}^{2} \int d m_{\pi \pi}^{2}\left|F_{\tilde{D} D}\right|^{2} \\
\quad \times\left[m_{\pi \pi}^{2}-4 M_{\pi}^{2}+\frac{1}{4 M_{\tilde{D}}^{2}}\left(m_{\pi \pi}^{2}-M_{\tilde{D}}^{2}-M_{D}^{2}-2 M_{\pi}^{2}+2 m_{D \pi}^{2}\right)^{2}\right] .
\end{aligned}
$$

In the present case, $\left.m_{\pi \pi}\right|_{\max } \simeq 560 \mathrm{MeV}$ is much larger than the two-pion threshold and hence the width becomes larger than other two-pion processes. We find

$$
\Gamma\left(\tilde{D}_{u, d}\left(1^{+}\right) \rightarrow D_{u, d}\left(0^{-}\right)+\pi^{ \pm} \pi^{0}\right)=314 \mathrm{keV} .
$$

Finally we turn to the decay $\tilde{D}_{s}\left(1^{+}\right) \rightarrow D_{s}\left(0^{-}\right)+\pi^{+} \pi^{-}$which as mentioned could receive direct contributions from scalar excitations. Since we have not incorporated scalar degrees of freedom in the theory, we might not be able to make a reliable estimate even if were to go to higher-loop orders. Just to have an idea as to how important the vector meson contribution can be, we calculate the decay width in which the $\tilde{D}_{s}$ meson decays into two pions through the $\phi$ meson. This isospin violating decay can occur through the direct $\phi-\pi-\pi$ coupling and the $\phi$ - $\rho$ mixing:

$$
\begin{aligned}
& \tilde{D}_{s}\left(1^{+}\right) \rightarrow D_{s}\left(0^{-}\right)+\left(\phi \rightarrow \pi^{+} \pi^{-}\right) \quad \text { (direct) } \\
& D_{s}\left(0^{-}\right)+\left(\phi \rightarrow \rho^{0} \rightarrow \pi^{+} \pi^{-}\right) \quad(\phi-\rho \text { mixing })
\end{aligned}
$$

Since the main contribution to the $\phi \rightarrow \pi \pi$ is expected to be given by the $\phi-\rho$ mixing, we shall neglect the direct $\phi-\pi-\pi$-coupling contribution in the following. Then the decay width is given by

$$
\begin{aligned}
\Gamma\left(\tilde{D}_{s}\left(1^{+}\right) \rightarrow D_{s}\left(0^{-}\right)+\pi^{+} \pi^{-}\right) \\
=\frac{M_{Q}^{2}}{192(2 \pi)^{3} M_{\tilde{D}}^{3}} \frac{k^{2}}{F_{\pi}^{4}} \int d m_{D \pi}^{2} \int d m_{\pi \pi}^{2}\left[\frac{M_{\rho}^{2} \Pi_{\phi \rho}}{\left(m_{\pi \pi}^{2}-M_{\phi}^{2}\right)\left(m_{\pi \pi}^{2}-M_{\rho}^{2}\right)}\right]^{2} \\
\quad \times\left[m_{\pi \pi}^{2}-4 M_{\pi}^{2}+\frac{1}{4 M_{\tilde{D}}^{2}}\left(m_{\pi \pi}^{2}-M_{\tilde{D}}^{2}-M_{D}^{2}-2 M_{\pi}^{2}+2 m_{D \pi}^{2}\right)^{2}\right]
\end{aligned}
$$

where $\Pi_{\phi \rho}$ denotes the $\phi$ - $\rho$ mixing given by

$$
\Pi_{\phi \rho}^{2}=\left(M_{\phi}^{2}-M_{\rho}^{2}\right)^{2}\left(\frac{\bar{p}_{\pi}(\rho)}{\bar{p}_{\pi}(\phi)}\right)^{3} \frac{M_{\phi}^{2}}{M_{\rho}^{2}} \frac{\Gamma\left(\phi \rightarrow \pi^{+} \pi^{-}\right)}{\Gamma\left(\rho \rightarrow \pi^{+} \pi^{-}\right)},
$$

with $\bar{p}_{\pi}(X)$ being the three-momentum of pion in the rest frame of the decaying particle $X=\phi, \rho$. Using the values listed in Table 【, we have

$$
\Pi_{\phi \rho}=530(\mathrm{MeV})^{2}
$$




\begin{tabular}{|c|c|c|}
\hline Decaying particle & Process & Width $(\mathrm{MeV})$ \\
\hline \multirow[t]{7}{*}{$\tilde{D}_{u, d}$} & $0^{+} \rightarrow 0^{-}+\pi^{0}$ & $7.37 \times 10^{1}$ \\
\hline & $0^{+} \rightarrow 0^{-}+\pi^{ \pm}$ & $1.47 \times 10^{2}$ \\
\hline & $0^{+} \rightarrow 1^{-}+\pi^{ \pm} \pi^{0}$ & $1.54 \times 10^{-5}$ \\
\hline & $1^{+} \rightarrow 1^{-}+\pi^{0}$ & $5.72 \times 10^{1}$ \\
\hline & $1^{+} \rightarrow 1^{-}+\pi^{ \pm}$ & $1.14 \times 10^{2}$ \\
\hline & $1^{+} \rightarrow 0^{-}+\pi^{ \pm} \pi^{0}$ & $3.14 \times 10^{-1}$ \\
\hline & $1^{+} \rightarrow 1^{-}+\pi^{ \pm} \pi^{0}$ & $1.18 \times 10^{-2}$ \\
\hline \multirow[t]{4}{*}{$\tilde{D}_{s}$} & $0^{+} \rightarrow 0^{-}+\pi^{0}$ & $4.17 \times 10^{-3}$ \\
\hline & $1^{+} \rightarrow 0^{+}+\pi^{0}$ & $1.87 \times 10^{-6}$ \\
\hline & $1^{+} \rightarrow 1^{-}+\pi^{0}$ & $3.75 \times 10^{-3}$ \\
\hline & $1^{+} \rightarrow 0^{-}+\pi^{+} \pi^{-} \quad\left(\right.$ through $\left.\phi \rightarrow \rho^{0} \rightarrow \pi^{+} \pi^{-}\right)$ & $2.13 \times 10^{-7}$ \\
\hline
\end{tabular}

TABLE II: The predicted values of the hadronic decay processes.

so the decay width is predicted to be

$$
\Gamma\left(\tilde{D}_{s}\left(1^{+}\right) \rightarrow D_{s}\left(0^{-}\right)+\pi^{+} \pi^{-}\right)=2.13 \times 10^{-4} \mathrm{keV} .
$$

The $\phi-\rho$ mixing is caused by the isospin violation, and this process is highly suppressed. We conclude that should a measured width come out to be substantially greater than what we found here, it would mean that either scalars must figure importantly or the VM is invalid in its present form.

\subsection{Summary of hadronic decay modes}

Our predictions of the decay widths are summarized in Table III. It should be stressed that the values obtained in this paper on the one-pion reflect only that the $\tilde{D}$ meson is the chiral partner of the $D$ meson. They are not specific to the VM. We therefore expect that as far as the one-pion processes are concerned, there will be no essential differences between our predictions and those in Ref. [28]. However, in the two-pion decay processes in which the scalar meson does not mediate, our scenario based on the VM can make definite predictions which might be distinguished from that based on the standard picture. Especially for $\tilde{D}_{u, d}\left(1^{+}\right) \rightarrow$ $D_{u, d}\left(0^{-}\right)+\pi^{ \pm} \pi^{0}$, we obtain a larger width than for other two-pion modes. Although the predicted width is still small - perhaps too small to be detected experimentally, it is important because of the following reason. In our approach, since the excited heavy meson multiplets of 
$\tilde{D}\left(0^{+}\right)$and $\tilde{D}\left(1^{+}\right)$denoted by $G$ are the chiral partners to the ground-state multiplets denote by $H$, the $G-\bar{H}-\pi$ coupling is the same as the $H-\bar{H}-\pi$ coupling [see the fifth and first terms of Eq. (2.29)]. Thus the width which is dependent on the strength of $k$ is a good probe to test our scenario. The common $k$ is also essential for the ratio of the widths of the two-pion modes to those of the one-pion modes, which has no $k$ dependence. These are therefore are definite predictions of our scenario. From the values listed in Table [II we obtain

$$
\frac{\Gamma\left(\tilde{D}_{u, d}\left(1^{+}\right) \rightarrow D_{u, d}\left(0^{-}\right)+\pi^{ \pm} \pi^{0}\right)}{\Gamma_{\pi+2 \pi}^{(\mathrm{had})}}=1.83 \times 10^{-3},
$$

where $\Gamma_{\pi+2 \pi}^{(\mathrm{had})}$ is the sum of the widths of the one-pion and two-pion modes of the decaying $\tilde{D}_{u, d}\left(1^{+}\right)$.

\section{SUMMARY AND DISCUSSIONS}

Let us summarize what we have accomplished in this paper. In Ref. [6], it was suggested that the chiral doubling of the heavy-light mesons could be exploited as a litmus indicator for chiral symmetry restoration by measuring the splitting at high temperature or density. If the splitting is indeed tied to the quark condensate which is an order parameter of chiral symmetry, one could observe the splitting disappearing at the critical point $C_{\chi}$. In this paper, we go the other way around. We start by the observation that at the critical point, the vector manifestation (VM) is realized [9, 11] so that hadron masses vanish in a manner predicted by BR scaling [14]. By introducing the deviation from the VM fixed point in terms of chiral symmetry breaking in the heavy-light system and matching the EFT so constructed to QCD at the matching scale, the "bare" mass splitting of the chiral doublers is determined in terms of the quark condensate and other QCD parameters of the system. The physical splitting is then determined by doing renormalization group evolution of the parameters with the bare Lagrangian matched to QCD. It is found to reproduce semi-quantitatively - modulo the spin assignments - the observed splitting which is related to the constituent quark mass. This result suggests rather strongly that identifying the chiral restoration as the VM fixed point and the chiral doubling as a signal of spontaneous breaking of chiral symmetry are mutually consistent.

One of the significant results of the analysis presented in this paper is that the vector meson plays an important role in accounting for the splitting in the $D$ and $\tilde{D}$ mesons: The bare mass splitting determined through the matching is estimated as about $190 \mathrm{MeV}$, too small to explain the observed mass difference. However by including the quantum corrections through the hadronic loop, the bare mass splitting is enhanced by $\sim 60 \%$, where only the loop effect of 
the vector meson contributes to the running of the mass splitting. The contributions from the pion loop are completely cancelled among themselves. This implies that the observed mass difference can not be understood if one takes only the pion as the relevant degree of freedom and that we need other degrees of freedom. In the VM, it is nothing but the vector meson. The situation here is much like in the calculation of pion velocity at the chiral restoration point: The pion velocity is zero if the pion is the only effective degree of freedom but approaches 1 if the vector meson with the VM is included [15].

Moreover, the result is independent of the deviation of $a$ from the fixed point value 1 at one-loop level. In other word, the resultant form of the quantum correction at one-loop level is completely independent of $a$. This implies that the deviation of $a$ from 1 which reflects the effect of spontaneous chiral symmetry breaking in the light quark sector does not get transmitted to the heavy sector. This strongly suggests that the deviation from $a=1$ involves physics that is not as primary as the non-vanishing gauge coupling $g \neq 0$ in the description of the broken phase: The deviation seems to be a "secondary" phenomenon, which is generated from $g \neq 0$ as expected in Refs. [32, 33]. "\#12 In fact, even when we start from the HLS theory with $g \neq 0$ and $a=1$, the physical quantities obtained through the Wilsonian matching are in good agreement with experimental results as discussed in [9]. This observation supports the above argument. It is intriguing to note that $a \sim 1$ is realized in the structure of both non-exotic and exotic baryons such as the nucleon electromagnetic form factor [35] and the skyrmion description of the $\Theta^{+}$pentaquark [36].

In section [6 we studied the hadronic decay processes of the $\tilde{D}$ mesons and showed the predictions of our scenario. The predictions on the one-pion processes are the consequences of the fact that the $\tilde{D}$ meson is the chiral partner to the $D$ meson, and there are no essential differences between our predictions and those in Ref. [28]. On the other hand, in the twopion decay processes in which the scalar meson does not mediate, our scenario gives definite predictions, since the vector meson coupling to the heavy system is equivalent to the pion coupling due to the VM. Although the predicted values of widths are small, we hope that they are clarified in future experiment.

Several comments are in order:

In this paper, we introduced spontaneous chiral symmetry breaking in the heavy sector by $\Delta M_{\text {bare }}$ only. Although we took the common coefficient $k$ for all the interaction terms in Eq. (2.29), each interaction term generally has its own coefficient different from others. However, we expect that the effect of these interaction terms is suppressed by the factor $1 / \Lambda$

\#12 Although $a=1$ is the fixed point of the RGE at one-loop level, the deviation of $a$ from 1 is generated by the finite renormalization part once we allow the deviation of the gauge coupling $g$ from 0 [34]. 
and as a result the contribution to $\Delta M$ is small since the dimension of them is higher than that of the mass term.

It is interesting that the bare splitting depends on the heavy-meson decay constant. This suggests that the splitting may show heavy-quark flavor dependence. This could be checked with experiments once a systematic heavy-quark expansion (which is not done here) is carried out. It is only in this sense that (part of) the splitting can be identified with the light-quark constituent mass discussed in Ref. [1, 2].

\section{Acknowledgment}

One of the authors (MR) is grateful for illuminating discussions with Maciek Nowak. MH and CS would like to thank the members of Korea Institute for Advanced Study (KIAS) for the warm hospitality where this work was partially done. The work of MH and CS are supported in part by the 21st Century COE Program of Nagoya University provided by Japan Society for the Promotion of Science (15COEG01), and by the JSPS Grant-in-Aid for Scientific Research (c) (2) 16540241 .

\section{APPENDIX A: EXPLICIT CALCULATION OF QUANTUM CORRECTION}

In this appendix, we compute the quantum effects on the masses of $0^{-}(P)$ and $0^{+}\left(Q^{*}\right)$ heavy-light $\mathcal{M}$-mesons by calculating the one-loop corrections to the two-point functions of $P$ and $Q^{*}$ denoted by $\Pi_{P P}$ and $\Pi_{Q^{*} Q^{*}}$. Here we adopt the following regularization method to identify the power divergences: We first perform the integration over the temporal component of the integration momentum, and then in the remaining integration over three-momentum we make the replacements given by

$$
\int^{\Lambda} \frac{d^{3} \vec{k}}{(2 \pi)^{3}} \frac{1}{\bar{k}^{2}} \rightarrow \frac{\Lambda}{2 \sqrt{2} \pi^{2}}, \quad \int^{\Lambda} \frac{d^{3} \vec{k}}{(2 \pi)^{3}} \frac{1}{\bar{k}} \rightarrow \frac{\Lambda^{2}}{8 \pi^{2}}, \quad \int^{\Lambda} \frac{d^{3} \vec{k}}{(2 \pi)^{3}} \rightarrow \frac{\Lambda^{3}}{12 \sqrt{2} \pi^{2}} .
$$

Here we use the t'Hooft-Feynman gauge for fixing the gauge of the HLS.

The diagrams contributing to $\Pi_{P P}$ are shown in Fig. 2. In the limit of zero external momentum, the divergent parts of these contributions are given by

$$
\begin{aligned}
& \left.\Pi_{P P}^{(a)[\sigma P]}\right|_{\text {div }}=\frac{2 k^{2}}{F_{\sigma}^{2}}\left[-\frac{M_{H}}{(4 \pi)^{2}}\left(\Lambda^{2}-2 M_{\rho} \ln \Lambda\right)+\frac{M_{H}^{2}}{4 \pi^{2}}\left(\frac{\Lambda}{\sqrt{2}}-M_{H} \ln \Lambda\right)\right], \\
& \left.\Pi_{P P}^{(b)\left[\pi P^{*}\right]}\right|_{\text {div }}=\frac{2 k^{2}}{F_{\pi}^{2}}\left[\frac{\Lambda^{3}}{24 \sqrt{2} \pi^{2}}-\frac{M_{H}}{(4 \pi)^{2}} \Lambda^{2}+\frac{M_{H}^{2}}{4 \pi^{2}}\left(\frac{\Lambda}{\sqrt{2}}-M_{H} \ln \Lambda\right)\right], \\
& \left.\Pi_{P P}^{(c)[\rho P]}\right|_{\text {div }}=\frac{g^{2}}{2 \pi^{2}}(1-k)^{2}\left(\frac{\Lambda}{\sqrt{2}}-M_{H} \ln \Lambda\right),
\end{aligned}
$$




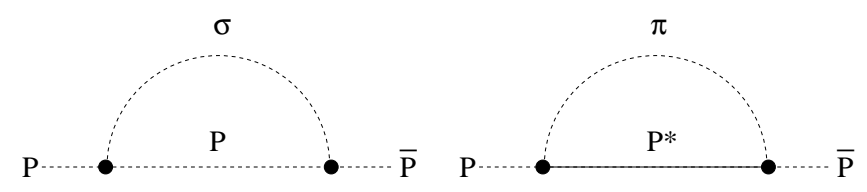

(a)

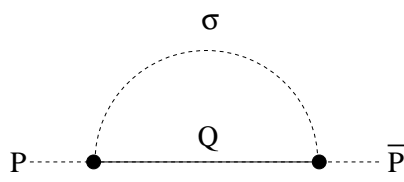

(d) (b)

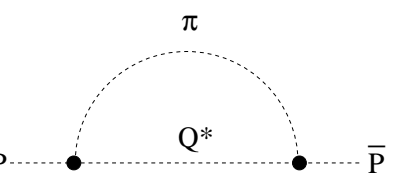

(e)

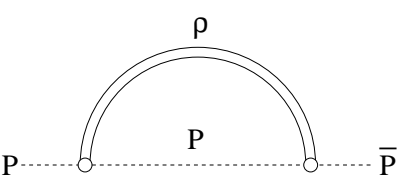

(c)

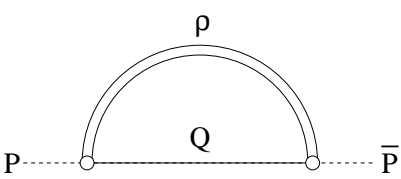

(f)

FIG. 2: Diagrams contributing to $P-P$ two point function.

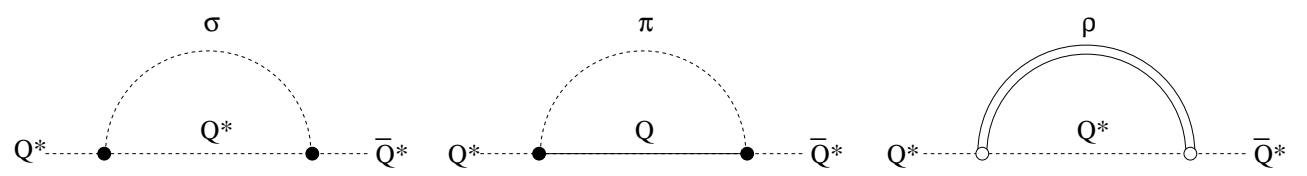

(a)

(b)

(c)

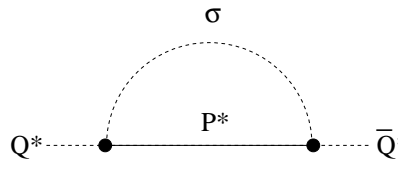

(d)

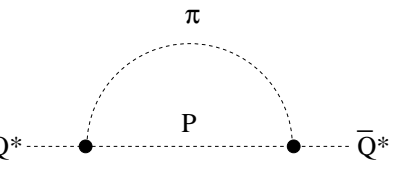

(e)

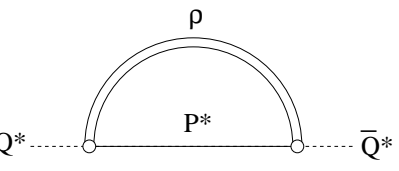

(f)

FIG. 3: Diagrams contributing to $Q^{*}-Q^{*}$ two point function.

$$
\begin{aligned}
& \left.\Pi_{P P}^{(d)[\sigma Q]}\right|_{\text {div }}=\frac{2 k^{2}}{F_{\sigma}^{2}}\left[\frac{\Lambda^{3}}{24 \sqrt{2} \pi^{2}}-\frac{M_{G}}{(4 \pi)^{2}}\left(\Lambda^{2}-2 M_{\rho}^{2} \ln \Lambda\right)+\frac{M_{G}^{2}-M_{\rho}^{2}}{4 \pi^{2}}\left(\frac{\Lambda}{\sqrt{2}}-M_{G} \ln \Lambda\right)\right] \\
& \left.\Pi_{P P}^{(e)\left[\pi Q^{*}\right]}\right|_{\text {div }}=\frac{2 k^{2}}{F_{\pi}^{2}}\left[-\frac{M_{G}}{(4 \pi)^{2}} \Lambda^{2}+\frac{M_{G}^{2}}{4 \pi^{2}}\left(\frac{\Lambda}{\sqrt{2}}-M_{G} \ln \Lambda\right)\right] \\
& \left.\Pi_{P P}^{(f)[\rho Q]}\right|_{\text {div }}=\frac{3 g^{2}}{2 \pi^{2}} k^{2}\left(\frac{\Lambda}{\sqrt{2}}-M_{G} \ln \Lambda\right) .
\end{aligned}
$$

The particles that figure in the loop are indicated by the suffix in square bracket; e.g., $\left[\pi P^{*}\right]$ indicates that $\pi$ and $P^{*}$ enter in the internal lines. Here and henceforth, we suppress, for notational simplification, the group factor $\mathcal{C}_{2}\left(N_{f}\right)$ defined as $\left(T_{a}\right)_{i j}\left(T_{a}\right)_{j l}=\mathcal{C}_{2}\left(N_{f}\right) \delta_{i l}$.

The relevant diagrams contributing to $\Pi_{Q^{*} Q^{*}}$ are shown in Fig. 3. The divergent parts of these contributions in the low-energy limit are expressed as

$$
\begin{aligned}
& \left.\Pi_{Q^{*} Q^{*}}^{(a)\left[\sigma Q^{*}\right]}\right|_{\text {div }}=\frac{2 k^{2}}{F_{\sigma}^{2}}\left[-\frac{M_{G}}{(4 \pi)^{2}}\left(\Lambda^{2}-2 M_{\rho}^{2} \ln \Lambda\right)+\frac{M_{G}^{2}}{4 \pi^{2}}\left(\frac{\Lambda}{\sqrt{2}}-M_{G} \ln \Lambda\right)\right], \\
& \left.\Pi_{Q^{*} Q^{*}}^{(b)[\pi Q]}\right|_{\text {div }}=\frac{2 k^{2}}{F_{\pi}^{2}}\left[\frac{\Lambda^{3}}{24 \sqrt{2} \pi^{2}}-\frac{M_{G}}{(4 \pi)^{2}} \Lambda^{2}+\frac{M_{G}^{2}}{4 \pi^{2}}\left(\frac{\Lambda}{\sqrt{2}}-M_{G} \ln \Lambda\right)\right],
\end{aligned}
$$




$$
\begin{aligned}
& \Pi_{Q^{*} Q^{*}}^{\left.(c)\left[\rho Q^{*}\right]\right|_{\text {div }}}=\frac{g^{2}}{2 \pi^{2}}(1-k)^{2}\left(\frac{\Lambda}{\sqrt{2}}-M_{G} \ln \Lambda\right), \\
& \left.\Pi_{Q^{*} Q^{*}}^{(d)\left[\sigma P^{*}\right]}\right|_{\text {div }}=\frac{2 k^{2}}{F_{\sigma}^{2}}\left[\frac{\Lambda^{3}}{24 \sqrt{2} \pi^{2}}-\frac{M_{H}}{(4 \pi)^{2}}\left(\Lambda^{2}-2 M_{\rho}^{2} \ln \Lambda\right)+\frac{M_{H}^{2}-M_{\rho}^{2}}{4 \pi^{2}}\left(\frac{\Lambda}{\sqrt{2}}-M_{H} \ln \Lambda\right)\right] \\
& \left.\Pi_{Q^{*} Q^{*}}^{(e)[\pi P]}\right|_{\text {div }}=\frac{2 k^{2}}{F_{\pi}^{2}}\left[-\frac{M_{H}}{(4 \pi)^{2}} \Lambda^{2}+\frac{M_{H}^{2}}{4 \pi^{2}}\left(\frac{\Lambda}{\sqrt{2}}-M_{H} \ln \Lambda\right)\right] \\
& \Pi_{Q^{*} Q^{*}}^{\left.(f)\left[\rho P^{*}\right]\right|_{\text {div }}}=\frac{3 g^{2}}{2 \pi^{2}} k^{2}\left(\frac{\Lambda}{\sqrt{2}}-M_{H} \ln \Lambda\right) .
\end{aligned}
$$

Now, let us compute the difference of $\Pi_{Q^{*} Q^{*}}-\Pi_{P P}$.

It is easy to show that $\left.\Pi_{P P}^{(b+e)}\right|_{\text {div }}$ exactly cancels with $\left.\Pi_{Q^{*} Q^{*}}^{(b+e)}\right|_{\text {div }}$. From the explicit forms given in Eqs. (A.2) and (A.3), we have

$$
\begin{aligned}
& \Pi_{Q^{*} Q^{*}}^{(b)[\pi Q]}-\left.\Pi_{P P}^{(e)\left[\pi Q^{*}\right]}\right|_{\mathrm{div}}=\frac{2 k^{2}}{F_{\pi}^{2}} \frac{\Lambda^{3}}{24 \sqrt{2} \pi^{2}}, \\
& \Pi_{Q^{*} Q^{*}}^{(e)[\pi P]}-\left.\Pi_{P P}^{(b)\left[\pi P^{*}\right]}\right|_{\mathrm{div}}=-\frac{2 k^{2}}{F_{\pi}^{2}} \frac{\Lambda^{3}}{24 \sqrt{2} \pi^{2}} .
\end{aligned}
$$

Note that the logarithmic, linear and quadratic divergences in $\Pi_{Q^{*} Q^{*}}$ are exactly canceled by those in $\Pi_{P P}$. This cancellation simply reflects that the external particles are chiral partners. This immediately leads to

$$
\Pi_{Q^{*} Q^{*}}^{(b+e)}-\left.\Pi_{P P}^{(b+e)}\right|_{\text {div }}=0
$$

The cubic divergence in $\Pi_{Q^{*} Q^{*}}$ is exactly canceled by that in $\Pi_{P P}$, reflecting that the internal particles are chiral partners to each other.

In a similar way, a partial cancellation takes place between $\Pi_{Q^{*} Q^{*}}^{(a)}$ and $\Pi_{P P}^{(d)}$ as well as between $\Pi_{Q^{*} Q^{*}}^{(d)}$ and $\Pi_{P P}^{(a)}$ :

$$
\begin{aligned}
& \Pi_{Q^{*} Q^{*}}^{(a)\left[\sigma Q^{*}\right]}-\left.\Pi_{P P}^{(d)[\sigma Q]}\right|_{\text {div }}=\frac{2 k^{2}}{F_{\sigma}^{2}}\left[-\frac{\Lambda^{3}}{24 \sqrt{2} \pi^{2}}+\frac{M_{\rho}^{2}}{4 \pi^{2}}\left(\frac{\Lambda}{\sqrt{2}}-M_{G} \ln \Lambda\right)\right] \\
& \Pi_{Q^{*} Q^{*}}^{(d)\left[\sigma P^{*}\right]}-\left.\Pi_{P P}^{(a)[\sigma P]}\right|_{\mathrm{div}}=\frac{2 k^{2}}{F_{\sigma}^{2}}\left[\frac{\Lambda^{3}}{24 \sqrt{2} \pi^{2}}-\frac{M_{\rho}^{2}}{4 \pi^{2}}\left(\frac{\Lambda}{\sqrt{2}}-M_{H} \ln \Lambda\right)\right] .
\end{aligned}
$$

These lead to

$$
\Pi_{Q^{*} Q^{*}}^{(a+d)}-\left.\Pi_{P P}^{(a+d)}\right|_{\text {div }}=-g^{2} \frac{k^{2}}{2 \pi^{2}}\left(M_{G}-M_{H}\right) \ln \Lambda
$$

where we used $M_{\rho}^{2}=g^{2} F_{\sigma}^{2}$. The remaining contributions sum to

$$
\Pi_{Q^{*} Q^{*}}^{(c+f)}-\left.\Pi_{P P}^{(c+f)}\right|_{\text {div }}=-g^{2} \frac{1-2 k-2 k^{2}}{2 \pi^{2}}\left(M_{G}-M_{H}\right) \ln \Lambda .
$$

By summing up the contributions in Eqs. (A.5), (A.7) and (A.8), we obtain the divergent part of the correction to the mass difference:

$$
\Pi_{Q^{*} Q^{*}}-\left.\Pi_{P P}\right|_{\mathrm{div}}=-\mathcal{C}_{2}\left(N_{f}\right) \frac{g^{2}}{2 \pi^{2}}\left(1-2 k-k^{2}\right)\left(M_{G}-M_{H}\right) \ln \Lambda
$$


where we reinstated the group factor $\mathcal{C}_{2}\left(N_{f}\right)$. The logarithmic divergence in the above expression is renormalized by the bare contribution given by

$$
\Pi_{Q^{*} Q^{*}}-\left.\Pi_{P P}\right|_{\text {bare }}=\Delta M_{\text {bare }}
$$

Thus the RGE for the mass difference $\Delta M=M_{G}-M_{H}$ has the following form:

$$
\mu \frac{d \Delta M}{d \mu}=\mathcal{C}_{2}\left(N_{f}\right) \frac{g^{2}}{2 \pi^{2}}\left(1-2 k-k^{2}\right) \Delta M .
$$

We should stress that this RGE is exactly the same as the one in Eq. (4.2) obtained by setting $a=1$, i.e., $F_{\sigma}=F_{\pi}$.

\section{APPENDIX B: NEED FOR A PRECISE VALUE OF THE QUARK CONDENSATE}

We emphasized in the main text that there is a great deal of uncertainty on the value of the quark condensate at the relevant matching scale $\Lambda_{M}$. In this Appendix, we list a few examples to show what sort of uncertainty we are faced with.

We took $\langle\bar{q} q\rangle_{1 \mathrm{GeV}}=-(225 \pm 25 \mathrm{MeV})^{3}$ in our analysis as a "standard value." For comparison,

we shall take two other values quoted in Ref. 23] (without making any judgments on their validity). Consider therefore

$$
\begin{aligned}
& \langle\bar{q} q\rangle_{1 \mathrm{GeV}}=-(225 \pm 25 \mathrm{MeV})^{3}, \\
& \langle\bar{q} q\rangle_{2 \mathrm{GeV}}=\left\{\begin{array}{l}
-(273 \pm 19 \mathrm{MeV})^{3} \\
-(316 \pm 24 \mathrm{MeV})^{3}
\end{array}\right.
\end{aligned}
$$

Brought by RGE to the scale we are working at, $\Lambda_{M}=1.1 \mathrm{GeV}$, and substituted into our formula for $\Delta M$, we get the corresponding quantum corrected splitting

$$
\Delta M=\left\{\begin{array}{l}
0.31 \pm 0.12 \mathrm{GeV} \\
0.43 \pm 0.12 \mathrm{GeV} \\
0.67 \pm 0.20 \mathrm{GeV}
\end{array}\right.
$$

This result clearly shows that the splitting cannot be pinned down unless one has a confirmed quark condensate. 
[1] M. A. Nowak, M. Rho and I. Zahed, Phys. Rev. D 48, 4370 (1993) arXiv:hep-ph/9209272.

[2] W. A. Bardeen and C. T. Hill, Phys. Rev. D 49, 409 (1994) arXiv:hep-ph/9304265.

[3] B. Aubert et al. [BABAR Collaboration], Phys. Rev. Lett. 90, 242001 (2003) arXiv:hep-ex/0304021.

[4] D. Besson et al. [CLEO Collaboration], Phys. Rev. D 68, 032002 (2003) arXiv:hep-ex/0305100.

[5] P. Krokovny et al. [Belle Collaboration], Phys. Rev. Lett. 91, 262002 (2003) arXiv:hep-ex/0308019.

[6] M. A. Nowak, M. Rho and I. Zahed, arXiv:hep-ph/0307102,

[7] M. Bando, T. Kugo, S. Uehara, K. Yamawaki and T. Yanagida, Phys. Rev. Lett. 54, 1215 (1985).

[8] M. Bando, T. Kugo and K. Yamawaki, Phys. Rept. 164, 217 (1988).

[9] M. Harada and K. Yamawaki, Phys. Rept. 381, 1 (2003) arXiv:hep-ph/0302103.

[10] M. Harada and K. Yamawaki, Phys. Rev. D 64, 014023 (2001) arXiv:hep-ph/0009163.

[11] M. Harada and K. Yamawaki, Phys. Rev. Lett. 86, 757 (2001).

[12] M. Harada and C. Sasaki, Phys. Lett. B 537, 280 (2002) arXiv:hep-ph/0109034.

[13] M. Harada, Y. Kim and M. Rho, Phys. Rev. D 66, 016003 (2002) arXiv:hep-ph/0111120.

[14] G. E. Brown and M. Rho, Phys. Rev. Lett. 66, 2720 (1991).

[15] C. Sasaki, Nucl. Phys. A739, 151 (2004); M. Harada, Y. Kim, M. Rho and C. Sasaki, Nucl. Phys. A730, 379 (2004).

[16] D. T. Son and M. A. Stephanov, Phys. Rev. D 66, 076011 (2002) arXiv:hep-ph/0204226.

[17] See, e.g., M. Asakawa, T. Hatsuda and Y. Nakahara, Nucl. Phys. A 715, 863 (2003) [Nucl. Phys. Proc. Suppl. 119, 481 (2003)] arXiv:hep-lat/0208059.

[18] See, e.g., , N. Arkani-Hamed, H. Georgi and M.D. Schwartz, Ann. Phys. 305, 96 (2003) arXiv:hep-th/0210184.

[19] S. Weinberg, Phys. Rev. 166, 1568 (1968).

[20] S. Narison, arXiv:hep-ph/0307248.

[21] S. Narison, Phys. Lett. B 210, 238 (1988).

[22] J. Gasser and H. Leutwyler, Phys. Rept. 87, 77 (1982).

[23] See, for a recent analysis, e.g., D. Becirevic and V. Lubicz, arXiv:hep-ph/0403044.

[24] B. Moussallam, Eur. Phys. J. C 14, 111 (2000) arXiv:hep-ph/9909292.

[25] K. Hagiwara et al. [Particle Data Group Collaboration], Phys. Rev. D 66, 010001 (2002).

[26] J. Schechter, A. Subbaraman and H. Weigel, Phys. Rev. D 48, 339 (1993) arXiv:hep-ph/9211239.

[27] M. Harada and J. Schechter, Phys. Rev. D 54, 3394 (1996) arXiv:hep-ph/9506473. 
[28] W. A. Bardeen, E. J. Eichten and C. T. Hill, Phys. Rev. D 68, 054024 (2003) arXiv:hep-ph/0305049.

[29] K. Abe et al. [Belle Collaboration], arXiv:hep-ex/0307021.

[30] P. Krokovny, arXiv:hep-ex/0310053.

[31] See, e.g., talk given by Y. J. Kwon [Belle Collaboration] at "2004 International Workshop on Hadron Physics: Pentaquark, Heavy-Light Hadrons and Hot/Dense Matter." (http://phya.yonsei.ac.kr/ pentaquark04/)

[32] H. Georgi, Phys. Rev. Lett. 63, 1917 (1989).

[33] H. Georgi, Nucl. Phys. B 331, 311 (1990).

[34] M. Harada and C. Sasaki, Nucl. Phys. A 736, 300 (2004) arXiv:hep-ph/0304282.

[35] G. E. Brown and M. Rho, Phys. Rept. 396, 1 (2004) arXiv:nucl-th/0305089.

[36] B. Y. Park, M. Rho and D. P. Min, arXiv:hep-ph/0405246. 